\title{
Intra-molecular origin of SNARF pH dependent fluorescence spectral properties
}

\begin{abstract}
Fluorescent molecules like SNARF are principally used to measure $\mathrm{pH}$ in liquid media. $\mathrm{pH}$ can be deduced from the fluorescence intensity or, often more precisely, by studying the fluorescence spectra. This allows numerical $\mathrm{pH}$ values to be calculated using some of the spectra shape features. Usually, the goal is to extract the contributions of the protonated and deprotonated forms of the $\mathrm{pH}$ indicator from the whole shape of the fluorescence spectrum and to deduce the $\mathrm{pH}$ value from these respective contributions. In this short paper, we show that analyzing the shape of the SNARF spectra differently allows specifying the intramolecular origin of the processes responsible for the fluorescence emission.
\end{abstract}

Keywords: fluorescence, $\mathrm{pH}$ sensing, SNARF, sensitive molecules, intracellular $\mathrm{pH}$, biological processes
Volume 6 Issue 4 - 2020

\author{
Bernard Gauthier-Manuel,' Rutjaphan \\ Kateklum,' Christian Pieralli,' Bruno \\ Wacogne ${ }^{1,2}$ \\ 'Univ. Bourgogne Franche-Comté, France \\ ${ }^{2}$ Besançon University Hospital, France
}

Correspondence: Bruno Wacogne, FEMTO-ST institut Univ. Bourgogne Franche-Comté, CNRS, I5B avenue des Montboucons, 25030 Besançon, cedex, France, Tel: + 33381666388, Fax + 33381666423,

Email bruno.wacogne@univ-fcomte.fr

Received: November 09, 2020 | Published: November 18, 2020

\begin{abstract}
Abbreviations:
SNARF, seminaphtharhodafluor benzenedicarboxylic acid, 2(or 4)-[10-(dimethylamino)-3-oxo$3 \mathrm{H}$-benzo[c]xanthene-7-yl]; FITC, fluorescein isothiocyanate 3',6'-dihydroxy-5(ou 6)-isothiocyanatospiro-3-one; BCECFAM, no common name found, 2',7'- bis-(carboxyethyl)-5(6')carboxyfluorescein acetoxymethyl ester, 2',7'-bis-(carboxyethyl)$5\left(6^{\prime}\right)$-carboxyfluorescein acetoxymethyl ester
\end{abstract}

\section{Introduction}

$\mathrm{pH}$ sensitive fluorescent molecules were largely synthetized in order to monitor $\mathrm{pH}$ in liquids and more particularly the intracellular $\mathrm{pH}$. The latter is related to the extracellular $\mathrm{pH}$ via ionic pumps. Dysregulation of these pumps directly affects biological processes. More generally, intracellular $\mathrm{pH}$ plays an important role in cells, enzymes and tissue activity like proliferation, apoptosis, resistance to drugs, ion transport or muscle contraction. Intracellular $\mathrm{pH}$ is also a key factor in many other biology fields which would be too long to present and outside the scope of this short paper. ${ }^{1}$ Using $\mathrm{pH}$ sensitive molecules, intracellular $\mathrm{pH}$ imaging is possible either in a direct fluorescence intensity imaging or ratiometric imaging techniques. For example, Hornig et $\mathrm{al}^{2}$ tested the diffusion of fluorescein and FITC in fibroblasts. ${ }^{2}$ Kaylor used SNARF in a ratiometric configuration at $570 \mathrm{~nm}$ and $620 \mathrm{~nm}$ in order to image tumors in mice, ${ }^{3}$ and found out that the $\mathrm{pH}$ was lower in the cancerous region. Gibbin and co-workers used the BCECF-AM pH indicator to monitor the effect of $\mathrm{CO}_{2}$ on intracellular $\mathrm{pH}$ with or without the use of a photosynthesis inhibitor in coral cells. ${ }^{4}$ Describing all the $\mathrm{pH}$ sensing molecules would be quite long and fastidious. Han and Burgess proposed a very complete review describing these molecules as described elsewhere. ${ }^{1}$ In this communication, we restrict our study to the case of the ratiometric indicator SNARF. SNARF exhibits a pKa equal to 7.5 which makes it a good candidate for sensing in the physiological $\mathrm{pH}$ range (about $\mathrm{pH}=6$ to 8 ). This molecule exhibits only two forms: protonated and deprotonated. Therefore, the fluorescence signal is due to two forms with relative contributions depending on the $\mathrm{pH}$ of the solution under test according to the law of mass action (see below). Each protonated or deprotonated form exhibits characteristic fluorescence and/or absorption spectra. ${ }^{5}$ Shifts between spectra obtained for protonated and deprotonated species can be exploited in order to perform a ratiometric measurement. In, ${ }^{5}$ discrepancies between $\mathrm{pH}$ measured using fluorescence and actual $\mathrm{pH}$ values were reported. This is mainly because not every proton participates in the acid-base equilibrium. Other works reported such discrepancies and attempts have been made to explain and/or correct them by mathematical means. ${ }^{6}$

Some authors developed mathematical models in order to account for different difficulties encountered in specific applications. For example in, ${ }^{7}$ authors developed a model to account for the small number of free $\mathrm{H}^{+}$ions in the yeast mitochondria. In reference ${ }^{6}$ authors study $\mathrm{pH}$ behavior in nanochannels. In this case, the zeta potential is responsible for charges reorganizations in the channels. Concentration of SNARF in these nanochannels is then influenced by the zeta potential which induces shifts in the measured $\mathrm{pH}$. Here, a model of the zeta potential was created and the indicator's concentration was included in this proposed model. $\mathrm{In}^{8}$ authors proposed to extend the 2 wavelengths ratiometric method to the analysis of the whole fluorescence spectrum. Their approach consisted in measuring and mathematically modeling the spectra of both fully protonated and fully deprotonated forms of SNARF. These two extreme $\mathrm{pH}$ spectra formed a basis which is now used to fit a spectrum recorded at an unknown $\mathrm{pH}$. Once the fitting is optimum, the value of the $\mathrm{pH}$ was determined. The same method was employed $\mathrm{in}^{9,10}$ with further consideration about the possibility to use SNARF to access the calibration free measurement. To summarize, in most cases, methods proposed to mathematically describe the fluorescence of ratiometric indicators are based on the decomposition of the spectrum in two components corresponding to extreme pHs spectra (acidic and basic). However, more information can be obtained from a mathematical analysis of the spectra shapes. In this paper, we show that describing SNARF emission spectra differently resulted in a schematically explanation of the intra-molecular photo-chemistry processes which are responsible for the $\mathrm{pH}$ dependent fluorescence of this molecule. By "intramolecular processes" we mean: parts of the molecule related to each individual fluorescence emission. In the next section, we briefly recall the fluorescence behavior of SNARF in terms of law of mass action. 
In addition, we show that $\mathrm{pH}$ dependent spectra can be efficiently described using only 3 gaussian functions with only a little number of $\mathrm{pH}$ dependent parameters. Moreover, we state a hypothesis in the explanation of the different parts of the SNARF molecule participated in the global $\mathrm{pH}$ dependent fluorescence spectra emission.

\section{Fluorescence properties of SNARF}

Fluorescent molecules like SNARF used for $\mathrm{pH}$ sensing can exist under protonated or deprotonated forms (Figure 1, note the "reaction site"). The deprotonation reaction of a dye is described as an equilibrium between these two forms.

$$
\text { DyeH } \leftrightharpoons D y e^{-}+H^{+}
$$

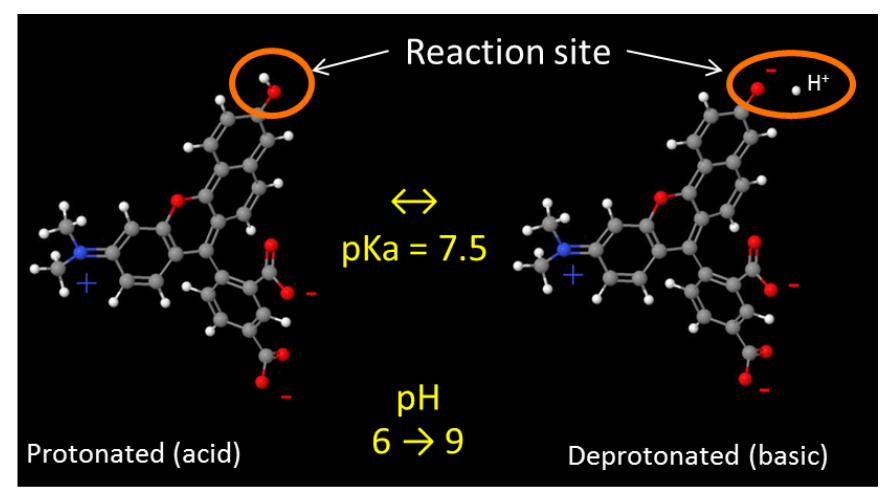

Figure I Representation of the deprotonation reaction of SNARF molecule. The $\mathrm{pKa}$ value is 7.5 which indicates an estimated $\mathrm{pH}$ sensitive range of the molecule between 6 and 9 . Grey = carbon, white = hydrogen, red = oxygen and blue $=$ nitrogen .

Where DyeH is the protonated form of the dye and Dye- the deprotonated form. Both forms exist simultaneously. Their respective concentrations are given by the law of mass action characterized by an equilibrium constant $\mathrm{K}$ function of the concentrations. This law allows defining a chemical equilibrium as a function of the species concentrations. It is related to the fact that the kinetic of a chemical reaction is proportional to the concentration of the different species. In our case, this law is written as follows.

$$
K_{a}=\frac{\left[D y e^{-}\right]\left[H^{+}\right]}{[D y e H]}
$$

In the same way as $\mathrm{pH}$ is related to the proton's activity, $\mathrm{pK}_{\mathrm{a}}$ is practically define as follows.

$$
p K_{a}=-\log _{10}\left(K_{a}\right)
$$

Mixing equations (2) and (3) gives the Henderson-Hasselbalch equation expressing the $\mathrm{pH}$ as a function of the $\mathrm{pK}_{\mathrm{a}}$ and species concentrations.

$$
p H=p K_{a}+\log _{10}\left(\frac{\left[D y e^{-}\right]}{[D y e H]}\right)
$$

From this, the dissociation constant is defined as follows.

$$
\alpha=\frac{1}{1+10^{\left(p K_{a}-p H\right)}}=\frac{1}{1+\left[H^{+}\right] / K_{a}}
$$

Finally, the concentrations of the protonated and deprotonated species can be expressed as a function of the total $\mathrm{pH}$ indicator concentration and dissociation constant as follows (for SNARF in this case).

$$
\begin{aligned}
& {\left[\text { SNARF }_{p}\right]=(1-\alpha)[S N A R F]} \\
& {\left[\text { SNARF }_{d}\right]=\alpha[\text { SNARF }]}
\end{aligned}
$$

Where $\left[S N A R F_{p}\right],\left[S N A R F_{d}\right]$ and $[S N A R F]$ are the concentrations of the protonated, deprotonated and total molecules. Figure 2 shows the fluorescence emission of SNARF. In Figure 2(B), we show the digitized data obtained from the supplier for $488 \mathrm{~nm}$ excitation wavelength. ${ }^{11}$ Figure 2(B) shows pictures of SNARF emission at extreme $\mathrm{pH}$ values with associated spectra. In what follows, we consider an excitation wavelength at $488 \mathrm{~nm}$.
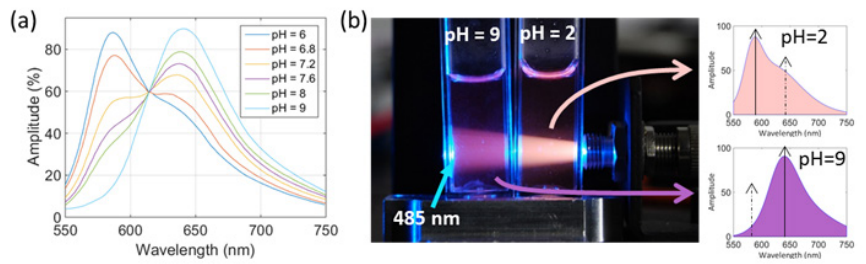

Figure 2 Fluorescence of SNARF. (A) Emission spectra obtained from the supplier ${ }^{12}$ for $488 \mathrm{~nm}$ excitation wavelength. (B) Spectra obtained at extreme $\mathrm{pH}$ values.

\section{Gaussian decomposition of fluorescence emission spectra (supplier's data)}

The general goal of the method consists in mathematically describe the $\mathrm{pH}$ dependent spectra of SNARF. For this, spectra obtained from the supplier are digitalized and processed as follows. For each of the $6 \mathrm{pH}$ values proposed by the supplier, we fit the spectra with a sum of "n" gaussian functions. Each individual spectrum is then decomposed in " $n$ " bands. The goal is to find the minimum number of bands required to fully describe spectra. Fitting spectra is performed in the wavenumbers domain. Therefore, in order to present equations with the traditional unit used in fluorescence $(\mathrm{nm})$, the gaussian function associated to the band number " $i$ " is written as follows.

$g_{i}(\lambda, p H)=A_{i}(p H) \exp \left[-\left(10^{7} \frac{\left(\frac{1}{\lambda}-\frac{1}{x_{i}(p H)}\right)}{2 \sigma_{i}(p H)}\right)^{2} \cdot 4 \log (2)\right] \quad i=1, n$

Here, $A_{i}(p H)$ is the $\mathrm{pH}$ dependent amplitude, $X_{i}(\mathrm{pH})$ the $\mathrm{pH}$ dependent position (in wavelength units) and $\sigma_{i}(\mathrm{pH})$ the $\mathrm{pH}$ dependent half-width (in wavenumber units) of gaussian function "i". After several fitting sessions, we found out that 3 gaussian functions fully describe the fluorescence spectra over the $\mathrm{pH}$ range provided by the supplier. This is illustrated in Figure 3. Band 1 (orange area in Figure 3) is extending over the whole spectral emission window. Functions $A_{1}(p H), X_{1}(\mathrm{pH})$ and $\sigma_{1}(p H)$ are represented in Figure 4. Their evolutions with $\mathrm{pH}$ are still not fully understood. Indeed, the behavior of band 1 with $\mathrm{pH}$ is probably due to the contribution of several internal molecular processes which are not yet identified. Values of the amplitudes, positions and half-widths of band 1 for the $\mathrm{pH}$ values obtained from the supplier are summarized in Table 1. Solid curves in Figure 4 correspond to fittings of parameters in Table 1 with 5th order polynomial functions in $\alpha$ (coefficients available on demand). Figure 5 shows a 3D view of the evolution of band 1 as a function of $\mathrm{pH}$ and wavelength. Evolutions of the last 2 bands with $\mathrm{pH}$ are much more informative. We determined that the centers and widths of these bands were constant. More remarkable, the amplitudes of these last 2 bands are exactly governed by the law of mass action described in equation (2) through the dissociation factor $\alpha$ (equation (5)). These 2 amplitudes are now written as follows and should be replaced in equations (8) for bands 2 and 3 respectively.

$$
\begin{gathered}
A_{2}=a_{2}(1-\alpha) \\
A_{3}=a_{3} \alpha
\end{gathered}
$$



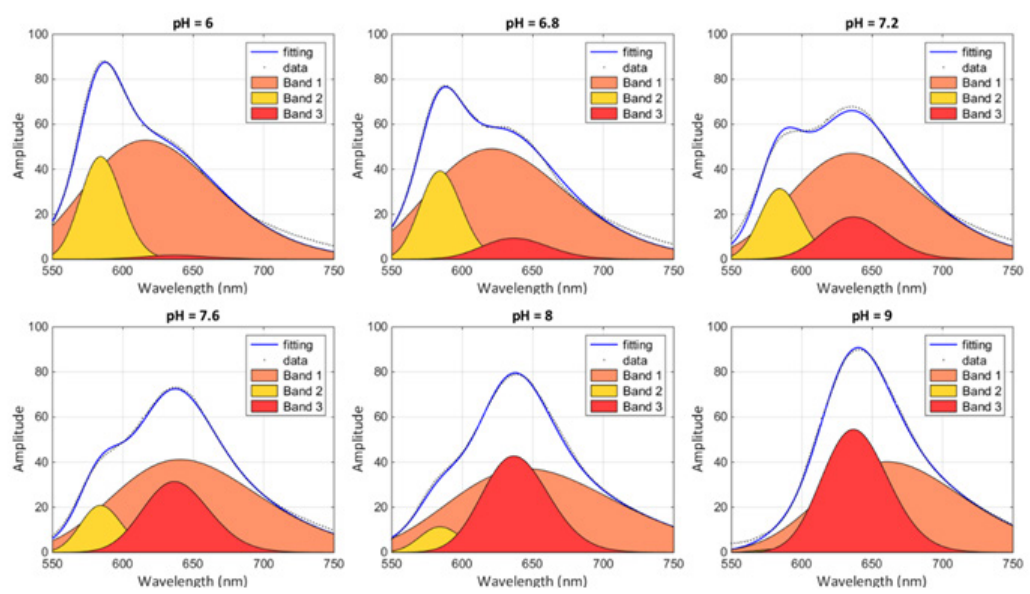

Figure 3 Fitting spectra obtained from supplier using 3 gaussian functions.
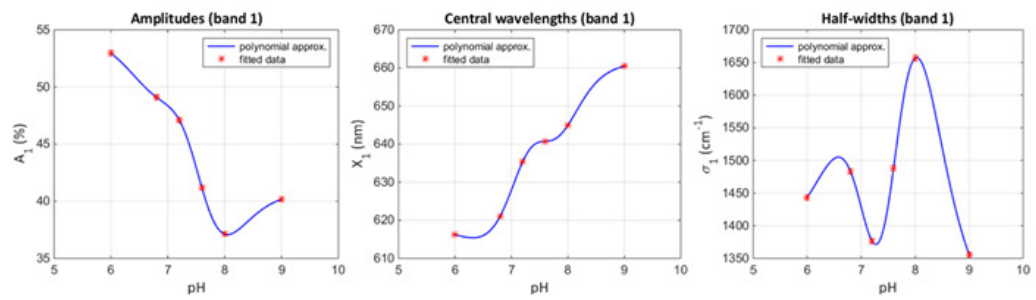

Figure 4 Evolution of the amplitude, position and half-width of band I with pH (spectra from supplier).

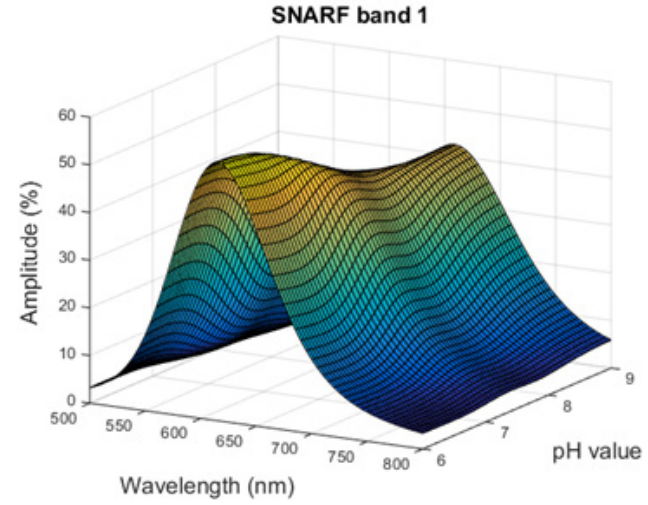

Figure 5 Band I as a function of $\mathrm{pH}$ and wavelength.

Here, $a_{2}$ and $a_{3}$ are the amplitudes of the last 2 gaussian functions at extreme $\mathrm{pH}$ values. They depended on the initial SNARF concentration and quantum parameters like the molar absorptivities, quantum yields of protonated and deprotonated forms and populations of the corresponding electronic levels. Figure 5 shows the 3D evolutions of bands 2 and 3 with $\mathrm{pH}$ and wavelength (Figure 6A) and a side view illustrating the evolutions of the amplitudes according to the law of mass action (Figure 6B). Parameters involved in equations (8) for bands 2 and 3 are summarized in Table 2.

\section{Gaussian decomposition from experimental data}

The goal of this section is to show that the gaussian decomposition is still valid when applied to home-obtained experimental data and to check whether or not bands 2 and 3 obey the law of mass action. Excitation was performed at $485 \mathrm{~nm}$ and the general experimental procedure is detailed below. Buffer solutions were Phosphate Buffer
Saline (PBS) 0.5X composed of $68.5 \mathrm{mM} \mathrm{NaCl}, 5 \mathrm{mM}$ phosphates ( $4 \mathrm{mM} \mathrm{Na}_{2} \mathrm{HPO}_{4}$ and $\mathrm{KH}_{2} \mathrm{PO}_{4}$ with proportion tuning the $\mathrm{pH}$ value), $1.35 \mathrm{mM} \mathrm{KCl}$.

1) $\mathrm{pHs}$ of buffer solutions were controlled with a Hanna instrument $\mathrm{pH} 211$, resolution 0.01 unit $\mathrm{pH}$.

2) SNARF (serial number 1270) was purchased from Thermofisher. A stock solution was prepared adding $1 \mathrm{mg}$ of indicator to $1 \mathrm{ml}$ of pure water. $40 \mu 1$ were sampled and added to $400 \mu 1$ of water to obtain aliquots used to prepare solutions of various $\mathrm{pHs}(20 \mu \mathrm{l}$ aliquot $+1 \mathrm{ml}$ buffer solution).

3) Plastic cuvettes purchased from Dominique Dutcher with $10 \mathrm{~mm}$ path length were filled with solutions at various $\mathrm{pHs}$ for fluorescence measurements.

4) Conventional $200 \mu \mathrm{m}$ core optical fibers purchased from Thorlabs (M38L01) were used to connect the excitation laser to the excitation port of the fluorescence beam-splitter and the emission port of the fluorescence beam-splitter to the spectrometer.

5) Spectra were acquired with a QEP00363 spectrometer purchased from Ocean Optics with an integration time of $100 \mathrm{~ms}$. Wavelength span $348 \mathrm{~nm}$ to $1127 \mathrm{~nm}, 1044$ pixels, and no boxcar or averaging were used.

Figure 7 shows the experimental set-up as well as the spectra recorded for several $\mathrm{pH}$ values. Spectra presented in Figure 7 underwent the same gaussian decomposition as spectra provided by the supplier. As it was the case with the supplier's data, the band 1 exhibits a not yet explained behavior with $\mathrm{pH}$ but the evolutions of the gaussian parameters are similar in both cases as depicted in Figure 8. Corresponding parameters are given in Table 3. Again, as was the case with spectra from supplier, bands 2 and 3 of our experimental 
data strictly obey the law of mass action through the dissociation constant. We don't present a Figure similar to Figure 3 in order not to lengthen the paper but corresponding coefficients are given in Table 4. To summarize this section, in both cases, bands 2 and 3 are defined by the law of mass action. The slight differences between data given in the Table are due to the fact that the compositions of the buffers we used were slightly different from the ones used by the manufacturer Spectra from supplier were obtained with $50 \mathrm{mM}$ potassium phosphate buffers while our spectra were obtained with buffers composition given above. Although of similar composition, these buffers are different in terms of buffer polarity. This is proven by the significant difference in emission spectra of the fluorophore. ${ }^{12}$ Because the buffer polarity modifies the emission spectrum of a fluorophore,,$^{12}$ values found in both cases slightly differ. However, because the law of mass action is obeyed in both cases for bands 2 and 3, we can state the following hypothesis.

\section{Intra-molecular origin of gaussian bands}

The hypothesis is based on the following observations.

- A gaussian fluorescence emission is due to a unique electronic transition. ${ }^{13}$

- 3 gaussian functions allow describing the fluorescence properties of SNARF.

- The spectra shapes of the protonated and deprotonated forms of SNARF are quite different with spectra maxima at about $580 \mathrm{~nm}$ and $640 \mathrm{~nm}$.

- This large Stokes shift between the protonated and the deprotonated forms shows that the presence or the absence of a proton has a strong impact on the energy levels inducing bands 2 and 3.

- Amplitudes of gaussian bands 2 and 3 exactly correspond to the law of mass action.

Table I Characteristics band I (spectra from supplier)

\begin{tabular}{llllllll}
\hline $\mathrm{pH}$ & $\mathbf{6}$ & $\mathbf{6 . 8}$ & $\mathbf{7 . 2}$ & $\mathbf{7 . 6}$ & $\mathbf{8}$ & $\mathbf{9}$ & Uncertainty \\
\hline $\boldsymbol{A}_{1}$ & 53.1 & 49.2 & 47.1 & 41.3 & 37.3 & 40 & $\pm 0.5 \%$ \\
$\boldsymbol{X}_{1}$ & 616.1 & 612 & 635.3 & 641.6 & 645.1 & 660.2 & $\pm 0.02 \%$ \\
$\boldsymbol{\sigma}_{1}$ & 1442 & 1476 & 1373 & 1484 & 1653 & 1355 & $\pm 0.3 \%$
\end{tabular}

Table 2 Parameters for bands 2 and 3 (spectra from supplier)

\begin{tabular}{llll}
\hline Coef. & Value & Uncertainty & Unit \\
$a_{2}$ & $47 . I \pm 0.2$ & $\pm 0.3 \%$ & $\%$ \\
$a_{3}$ & $56.3 \pm 0.4$ & $\pm 0.7 \%$ & $\%$ \\
$X_{2}$ & $584.2 \pm 0.3$ & $\pm 0.05 \%$ & $\mathrm{~nm}$ \\
$X_{3}$ & $636.6 \pm 0.5$ & $\pm 0.08 \%$ & $\mathrm{~nm}$ \\
$\sigma_{2}$ & $504 \pm 15$ & $\pm 3 \%$ & $\mathrm{~cm}^{-1}$ \\
$\sigma_{3}$ & $680 \pm 30$ & $\pm 5 \%$ & $\mathrm{~cm}^{-1}$ \\
\hline
\end{tabular}

Table 3 Characteristics band I (experimental data)

\begin{tabular}{llllllllll}
\hline $\mathbf{p H}$ & $\mathbf{6}$ & $\mathbf{6 . 4}$ & $\mathbf{6 . 8}$ & $\mathbf{7 . 2}$ & $\mathbf{7 . 6}$ & $\mathbf{8}$ & $\mathbf{8 . 5}$ & $\mathbf{9}$ & Uncertainty \\
\hline $\boldsymbol{A}_{1}$ & 14864 & 14685 & 14334 & 13575 & 13217 & 12465 & 12823 & $1289 \mid$ & $\pm 0.2 \%$ \\
$\boldsymbol{X}_{1}$ & 609 & 610 & 615 & 614 & 625 & 625 & 636 & 637 & $\pm 0.4 \%$ \\
$\sigma_{1}$ & 1519 & 1534 & 1533 & 1614 & 1589 & 1691 & 1590 & 1585 & $\pm 0.5 \%$ \\
\hline
\end{tabular}

Table 4 Parameters for bands 2 and 3 (experimental data)

\begin{tabular}{llll}
\hline Coef. & Value & Uncertainty & Unit \\
$a_{1}$ & $9932 \pm 40$ & $\pm 0.4 \%$ & Cts \\
$a_{2}$ & $13448 \pm 90$ & $\pm 0.68 \%$ & Cts \\
$X_{1}$ & $580.8 \pm 0.1$ & $\pm 0.02 \%$ & $\mathrm{~nm}$ \\
$X_{2}$ & $637 \pm 3$ & $\pm 0.5 \%$ & $\mathrm{~nm}$ \\
$\sigma_{1}$ & $564 \pm 5$ & $\pm 1.01 \%$ & $\mathrm{~cm}^{-1}$ \\
$\sigma_{2}$ & $638.59 \pm 0.06$ & $\pm 0.01 \%$ & $\mathrm{~cm}^{-1}$ \\
\hline
\end{tabular}

Therefore, bands 2 and 3 were resultant from the electronic transitions which occurred in a reduced region of the SNARF molecule, more likely within the reaction site shown in Figure 1. This is further illustrated in Figure 9 where the greenish regions correspond to bands 2 and 3. As previously stated, although well described by a gaussian function, band 1 cannot be directly related to a particular intra-molecular process. This is due to the fact that $\mathrm{pH}$ has a little effect of the behavior of band 1 as compared to its effect on band
2 and 3. The hypothesis is that it is generated in regions far away from the reaction site and defined by the reddish frontiers in Figure 9. However, although weak, the behavior of band 3 with respect to $\mathrm{pH}$ is still to be explained. Note that green and red regions in Figure 9 are drawn for illustration purposes. Their actual sizes and exact location of the frontier between them is not yet defined. In particular, we still don't know how many aromatic rings are involved in the behavior of bands 2 and 3 and how many are involved in the behavior of band 1 . 

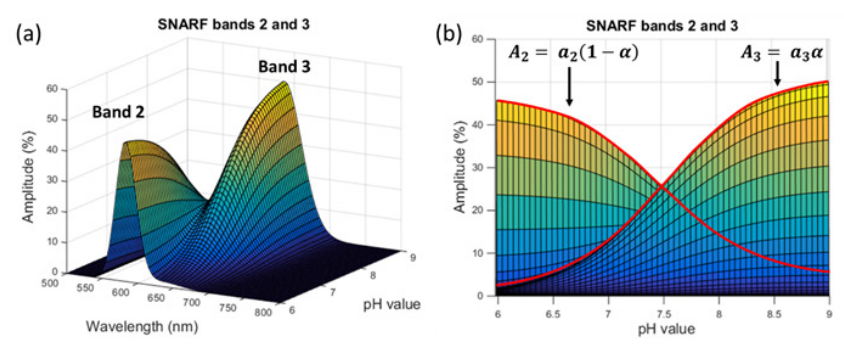

Figu

Figure 6 Bands 2 and 3. (A) 3D view of the evolution of bands 2 and 3 with $\mathrm{pH}$ and wavelength. (B) Side view showing the evolution of the amplitudes according to the law of mass action.
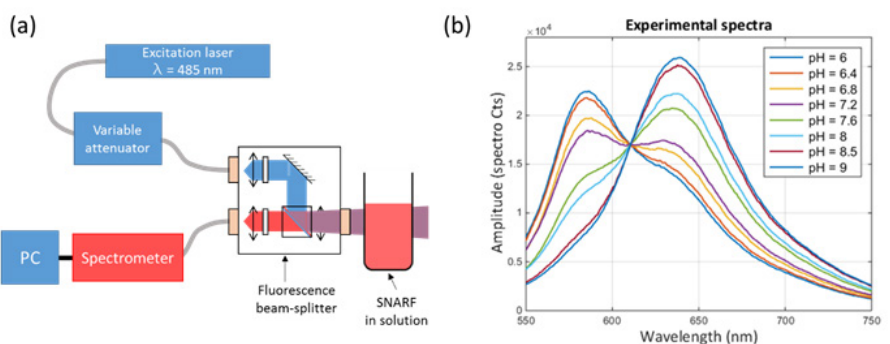

Figure 7 (A) Experimental set-up. (B) Experimental spectra with $\mathrm{pH}$ ranging from 6 to 9 .
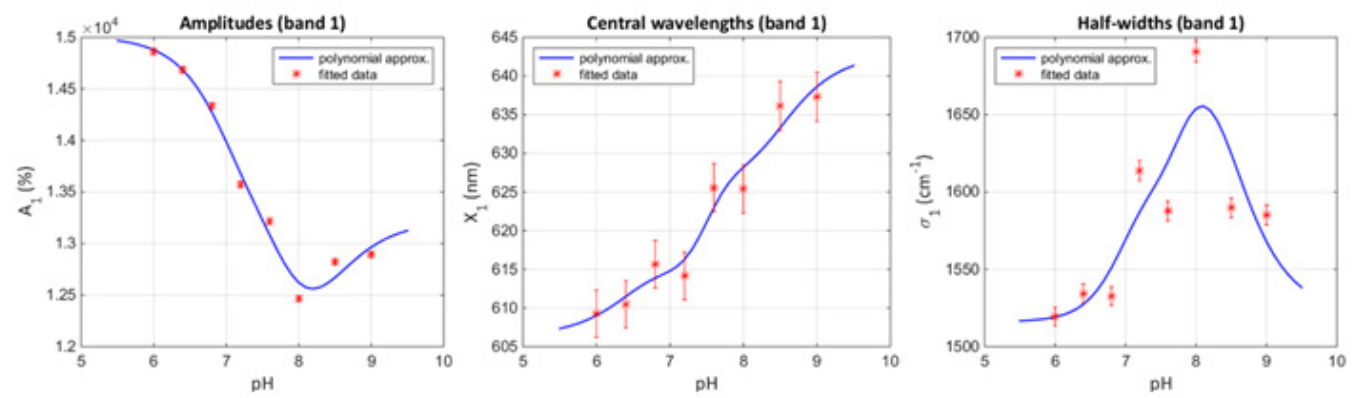

Figure 8 Evolution of the amplitude, position and half-width of band I with $\mathrm{pH}$ (experimental data).

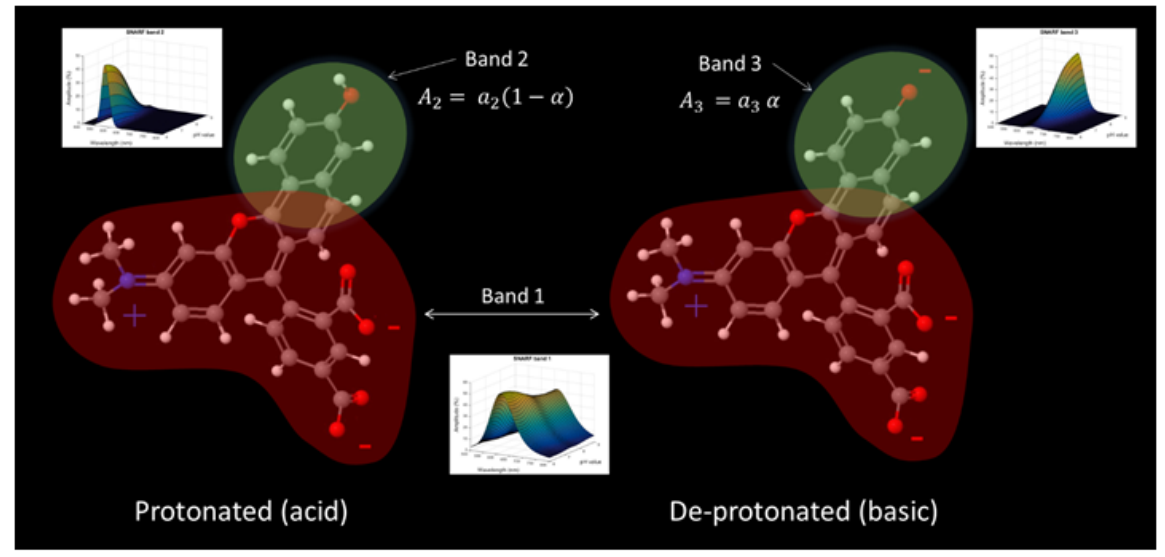

Figure $9 \mathrm{~A}$ tentative description of the intra-molecular origin of SNARF fluorescence behavior.

\section{Conclusion}

In this short paper, we mathematically investigated the $\mathrm{pH}$ dependent SNARF fluorescence properties. We showed that the $\mathrm{pH}$ dependent spectra can be described using only 3 gaussian functions. More interesting, we specified that the centers and widths of 2 of these gaussian profiles were constant. Slight differences between results obtained from the supplier's data and our experimental data are due to different buffer compositions. However, in both cases, the amplitudes of the 2 gaussian band with constant positions and widths strictly obey the law of mass action. The behavior of the other gaussian contribution is more difficult to explain. From this, we stated the hypothesis that a reduced intra-molecular region of the SNARF molecule, close to the place where protons are bounded or resealed, is involved in the production of 2 gaussian bands. The rest of the molecule is responsible for the last gaussian band and its lower $\mathrm{pH}$ dependence is due to the distance between this region and the proton reactive site of the molecule. Further investigations are still required to exactly define how many aromatic rings are involved in each of these bands.

\section{Acknowledgements}

This work was supported by the European Commission (grant number FE2007/2013, operation 36381).

\section{Conflicts of interest}

There is no conflict of interest.

\section{References}

1. Han J, Burgess K. Fluorescent Indicators for Intracellular Ph. Chem Rev. 2010;11(5):2709-2728. 
2. Hornig S, Biskup C, Gräfe A, et al. Biocompatible fluorescent nanoparticles for pH-sensoring. Soft Matter. 2008;4:1169-1172.

3. Kaylor BM, Gatenby RA, Gmitro AF. In Vivo Measurement of $\mathrm{pH}$ in Tumor and Surrounding Tissue using Fluorescence Ratio Imaging, Frontiers in Optics. OSA Technical Digest Series. 2005.

4. Gibbin EM, Putnam HM, Davy SK, et al. Intracellular $\mathrm{pH}$ and its response to $\mathrm{CO} 2$-driven seawater acidification in symbiotic versus nonsymbiotic coral cells. Journal of Experimental Biology. 2014;217:19631969.

5. Yassine M, Salmon JM, Vigoand J, et al. C-SNARF-1 as a pHi fluoroprobe: discrepancies between conventional and intracellular data do not result from protein interactions. Journal of Photochemistry and Photobiology B: Biology. 1997;37(1):18-25.

6. Bottenus D, Oh YJ, Han SM, et al. Experimentally and theoretically observed native $\mathrm{pH}$ shifts in a nanochannel array. Lab Chip. 2009;9:219231.

7. Żurawik TM, Pomorski A, BelczykCiesielska A, et al. Revisiting Mitochondrial $\mathrm{pH}$ with an Improved Algorithm for Calibration of the Ratiometric 5(6)-carboxy-SNARF-1 Probe Reveals Anticooperative Reaction with $\mathrm{H}+$ Ions and Warrants Further Studies of Organellar $\mathrm{pH}$. PLOS ONE. 2016;11(8):e0161353.
8. Ribou AC, Vigo J, Salmon JM. C-SNARF-1 as a fluorescent probe for $\mathrm{pH}$ measurements in living cells: two-wavelength-ratio method versus whole-spectral-resolution method. J of Chem Educ. 2002;79;(12):14711474.

9. Owen CS. Comparison of spectrum-shifting intracellular $\mathrm{pH}$ probes $5^{\prime}$ (and $\quad 6^{\prime}$ )-carboxy-10-dimethylamino-3-hydroxyspiro[7H-benzo[c] xanthene-7, 1'(3'H)-isobenzofuran]-3'-one and 2',7'-biscarboxyethyl5(and 6)-carboxyfluorescein. Analytical Biochemistry. 1992;204(1):6571.

10. Owen CS, Carango P, Grammer S, et al. pH-dependent intracellular quenching of the indicator carboxy-SNARF-1. Journal of fluorescence. 1992;2(2):75-80.

11. https://www.thermofisher.com/fr/fr/home/references/molecular-probesthe-handbook/ph-indicators/probes-useful-at-near-neutral-ph.html

12. Lakowicz JR. Principles of fluorescence spectroscopy. 3rd edn. Springer; 2006:205.

13. Heimel P, Mondal A, May F, et al. Unicolored phosphor-sensitized fluorescence for efficient and sTable blue OLEDs. Nat Commun. 2018;9:90. 